\author{
Organ der SMS, Societas Medicinae Sinensis \\ Internationale Gesellschaft für Chinesische Medizin e.V., \\ Franz-Joseph-Straße 38, D-80801 München \\ www.tcm.edu oder www.sms-tcm.de
}

Organ der SACAM, Schweizerische Ärztinnen- und Ärztegesellschaft für Akupunktur - Chinesische Medizin -

Aurikulomedizin

\section{Postfach 2003, CH-8021 Zürich, Schweiz}

www.sacam.ch

Die Zeitschrift Chinesische Medizin ist die erste deutschsprachige Zeitschrift, die sich nicht nur mit Akupunktur befasst, sondern auch mit den anderen Bereichen der chinesischen Medizin wie Arzneimitteltherapie, Diätetik, chinesische Bewegungstherapien (Taiji und Qigong) und chinesische manuelle Therapie (Tuina). Neben eigenen Beiträgen finden sich hier ausgesuchte Artikel aus den wichtigsten chinesischen Fachzeitschriften, die direkt aus dem Chinesischen ins Deutsche übersetzt werden.

Chinese Medicine is the official journal of the International Society for Chinese Medicine e.V. (SMS) and the Swiss Medical Society for Acupuncture - Chinese Medicine - Auriculomedicine (SACAM) and the first German-language journal to be devoted in depth to not only acupuncture but also the other aspects of Chinese medicine, e.g. pharmacology, dietetics, movement therapies (such as Taiji and Qigong) and Chinese manual therapy (Tuina). In addition to publishing articles by our own authors, we are the first Western journal to regularly publish selected articles from original Chinese medical journals translated directly into German.

\section{Herausgeber/Editors}

Dr. Rainer Nögel, München

Dr. Ute Engelhardt, München

Dr. Toni Fischer, Zürich

\section{Schriftleitung/Editor-in-Chief}

Dr. Ute Engelhardt (verantwortlich), Elisabethstraße 11,

D-80796 München, Telefon (+49/89) 27818539.

\section{Wissenschaftlicher Beirat/Advisory Board}

Dr. Mazin Al-Khafaji, Avicenna Centre of Chinese Medicine, England Prof. Dr. Rudolf Bauer, Institut für Pharmazeutische Wissenschaften der Karl-Franzens-Universität Graz

Prof. Dr. Benno Brinkhaus, Klinikum Charité der Humboldt-Universität zu Berlin

Prof. Catherine Despeux, Institut National des Langues et Civilisations Orientales (INALCO), Frankreich

Prof. Fang Chunyang, Zhejiang Academy of TCM, Hangzhou, China

Prof. Fan Jiayong, Chengdu University of TCM, China

Prof. Heiner Frühauf, National College of Natural Medicine, Portland/

Oregon, USA

Prof. Ingrid Gerhard, Universitäts-Frauenklinik Heidelberg

Prof. Dr. Carl-Hermann Hempen, TCM-Masterstudiengang an der

TU München

Prof. Livia Kohn, University of Boston, USA

Prof. Liang Fanrong, Chengdu University of TCM, China

Prof. Ma Lieguang, Chengdu University of TCM, China

Dr. C.L. Oei-Tan, Rotterdam, Niederlande

Prof. Dr. Claudia Witt, Institut für komplementäre und integrative

Medizin am UniversitätsSpital Zürich
Arzneimittel

141 Agenzien, Mechanismen und Behandlung des Sicca-Syndroms

Shen Pi'an

Akupunktur

156 Die Foramina Ex12/EX-B-2 (,,Zu beiden

Seiten der Wirbelsäule", huatuojiaji)

Agnes Fatrai, Rainer Nögel

Medizinhistorisches

163 Hua Tuo 華伦, ein sagenumwobener Arzt aus der Frühzeit Chinas

Ute Engelhardt

Akupunktur

169 Die Bedeutung der Ohrakupunktur bei der Behandlung des lumbalen Bandscheibenprolapses anhand von zwei Fallbeispielen Miriam Bernatik

182 Aus der Redaktion

188 Mitgliederinformationen

168 TCM-Quiz

181 Antworten TCM-Quiz

\section{jobcenter-medizin.de}

\title{
Cited in EMBASE/Excerpta Medica
}




\section{Chinesische Medizin}

Band $32 \cdot 2017$

\section{Contents}

Chinese Herbal Medicine

141 Agents, mechanisms and treatments of the Sicca Syndrome

Shen Pi'an

Acupuncture

156 The acupuncture points Ex12/EX-B-2 ("Hua Tuo's para-vertebral points", huatuojiaji) Agnes Fatrai, Rainer Nögel

History of Medicine

163 Hua Tuo 華佗 (approx. 141-203), a legendary physician in early China Ute Engelhardt

Acupuncture

169 The importance of auricular acupuncture in the treatment of prolapsed lumbar disc illustrated in two case studies

Miriam Bernatik

\section{From the Editor}

188 Members' Update

\section{TCM-quiz}

181 Answers to TCM-quiz

\author{
Verantwortliche Schriftleitung: Dr. Ute Engelhardt, Elisabethstraße 11, \\ D-80796 München \\ Verlag \\ Springer Medizin Verlag GmbH, Berlin \\ Ladungsfähige Anschrift und Kontaktdaten: \\ Springer Medizin Verlag GmbH, Aschauer Straße 30, 81549 München \\ Tel.:(089) 203043-1300, Fax: (089) 203043-1400 \\ www.SpringerMedizin.de \\ Geschäftsführer: Joachim Krieger, Fabian Kaufmann \\ Leitung wissenschaftliche Zeitschriften, München: Dr. Heidrun Guthöhrlein \\ Gesamtleitung Corporate Publishing: Ulrike Hafner \\ Anzeigenleitung: Michaela Schmitz \\ (Telefon 02203/95913-10, michaela.schmitz@springer.com) \\ Anzeigenpreisliste Nr. 30 vom 1.10.2015. \\ Herstellung: Ulrike Drechsler (Leitung) \\ Vertrieb: Frank Niemann \\ Druck \\ Ten Brink B.V., Eekhorstweg 1, 7942 JC Meppel, Niederlande
}

Erscheinungsweise: Vierteljährlich 1 Heft, 4 Hefte pro Jahrgang.

Bezugspreise inkl. Online-Basis-Lizenz 2017: Pro Jahrgang $€ 142,-$ (unverb. Preisempfehlung inkl. gesetzlicher MwSt.), Vorzugspreis für Ärzte in Aus- und Weiterbildung und Studenten: $€$ 85,20 (unverb. Preisempfehlung inkl. gesetzlicher MwSt.), jeweils zuzüglich Versandkosten (Inland $€ 21$,- Ausland $€ 30,-$ ). Einzelheft $€ 53$ - - (unverb. Preisempfehlung inkl. gesetzlicher MwSt.) zzgl. Versandkosten. Mitglieder der Societas Medicinae Sinensis Internationale Gesellschaft für Chinesische Medizin e.V. und der Schweizerischen Ärztegesellschaft für Akupunktur - Traditionelle Chinesische Medizin erhalten die Fachzeitschrift im Rahmen ihrer Mitgliedschaft. Der Bezugspreis ist im Mitgliedsbeitrag enthalten.

Allgemeiner Hinweis zur gesetzlichen Mehrwertsteuer: Gedruckte Zeitschriften unterliegen grundsätzlich dem ermäßigten Steuersatz von 7\%, digitale Produkte (wie z.B. die Online-Version einer Zeitschrift) hingegen dem allgemeinen Steuersatz von 19\%. Die detaillierte Aufteilung der einzelnen Mehrwertsteuer-Beträge entnehmen Sie bitte Ihrer Rechnung.

Bezugsmöglichkeiten: Bestellungen nehmen der Verlag und jede Buchhandlung entgegen. Abo-Service: Springer Customer Service Center GmbH: Tel. +49/6221 345-4304, Fax -4229. Das Abonnement verlängert sich jeweils um 1 Jahr, sofern nicht eine Abbestellung 30 Tage zum Ende des Bezugszeitraums erfolgt ist. Sollte die Fachzeitschrift aus Gründen, die nicht vom Verlag zu vertreten sind, nicht geliefert werden können, besteht kein Anspruch auf Nachlieferung oder Erstattung vorausgezahlter Bezugsgelder.

Handelsnamen und Dosierungen: Eine Markenbezeichnung kann warenzeichenrechtlich geschützt sein, auch wenn bei ihrer Verwendung in dieser Zeitschrift das Zeichen ${ }^{\circledR}$ oder ein anderer Hinweis auf etwa bestehende Schutzrechte fehlen sollte. Für Angaben über Dosierungsanweisungen und Applikationsformen kann vom Verlag keine Gewähr übernommen werden. Derartige Angaben müssen vom jeweiligen Anwender im Einzelfall anhand anderer Literaturstellen oder mit dem Beipackzettel des jeweiligen Medikaments, insbesondere bei Neuerscheinungen, verglichen werden.

Urheberrecht: Mit Annahme des Manuskripts zur Publikation erwirbt der Verlag das ausschließliche Nutzungsrecht, das die Erstellung von Fort- und Sonderdrucken, auch für Auftraggeber aus der Industrie, die Übersetzung in andere Sprachen, die Erteilung von Abdruckgenehmigungen für Teile, Abbildungen oder die gesamte Arbeit an andere Verlage sowie Nachdrucke in Zeitschriften des eigenen Verlags, die fotomechanische sowie elektronische Vervielfältigung und die Wiederverwendung von Abbildungen umfasst. Dabei ist die Quelle anzugeben. Änderungen und Hinzufügungen zu Originalpublikationen bedürfen der Zustimmung des Autors. Die Zeitschrift und alle in ihr enthaltenen Beiträge und Abbildungen sind urheberrechtlich geschützt. Jede Verwertung außerhalb der engen Grenzen des Urheberrechtsgesetzes ist ohne Zustimmung des Verlags unzulässig und strafbar. Das gilt insbesondere für Vervielfältigungen, Übersetzungen, Mikroverfilmungen und die Einspeicherung und Verarbeitung in elektronischen Systemen. (c) Springer Medizin Verlag $\mathrm{GmbH}$ ISSN 0930-2786

Informationen zur Zeitschrift online unter http://www.springermedizin.de 